\title{
Needle aspiration for suppurative post-BCG adenitis
}

\author{
S Abbas Banani, Abdolvahab Alborzi
}

\begin{abstract}
The effect of needle aspiration in suppurative post-BCG adenitis was studied. Nodes that had been aspirated (43 patients) regressed in $25(58 \%)$ and 41 $(95 \%)$ patients two and six months after aspiration. In the control group (34 patients) regression occurred in three $(9 \%)$ and $22(65 \%)$ patients. Spontaneous drainage with sinus tract formation was also significantly less in the aspirated group at six months ( $7 \% v \mathbf{v 4 \%}$ ).

(Arch Dis Child 1994; 71: 446-447)
\end{abstract}

Beside local ulceration and abscess formation at the site of vaccination, the most common complication of BCG vaccination is regional (axillary) adenitis. ${ }^{1}$ The involved nodes usually subside spontaneously, but they may become enlarged and form an abscess. Although different methods of management have been recommended, ${ }^{12}$ needle aspiration is rarely described as a mode of treatment in this situation. ${ }^{3}$ The role and value of needle aspiration in dealing with this complication is clarified in this controlled trial study.

\section{Patients and methods}

Between September 1991 and March 1993 referred infants or children with regional BCG adenitis were randomly assigned every other week into the study and control group. Standard Pasteur vaccine had been used in all cases $(0.05 \mathrm{ml}$ for neonates, $0.1 \mathrm{ml}$ if beyond 1 month). All patients except those with signs of abscess formation were evaluated sonographically for detection of any fluid in the node (presumably pus).

Patients with the following conditions were excluded: (a) no sonographic sign of node collection; (b) generalised adenopathy; (c) immunocomprised; (d) receiving an antituberculosis drug before referral. The study group was subjected to needle aspiration for complete evacuation of the pus but the patients in the control group were treated expectantly.

To avoid iatrogenic sinus formation, the aspiration was done subcutaneously $2-3 \mathrm{~cm}$ from the periphery of the node. The lesion was mildly compressed manually not only to facilitate evacuation, but to guide the needle in different directions (and to drain any collection in the adjacent nodes) without piercing the overlying skin.

Follow up visits continued every one to two months until the problem was either solved or stable, after which the patient was re-examined three to four months later. At each follow up visit, the node diameters were measured in two dimensions and reaspiration was performed if necessary. Any difference in consistency of the nodes or presence of a sinus tract was also noted. Yates's corrected $\chi^{2}$ and Fisher's exact tests were used for statistical analysis of the comparison of outcomes between the two groups.

\section{Results}

Seventy seven patients were included in this study: 43 in the study group, 34 in the control group. Nearly two thirds of the patients had been vaccinated in the first two weeks of life in both groups. The age at presentation was 1-36 months (mean for the study and control group were 10.4 and 10.8 months respectively).

In all except two patients in the study group the collection of pus was unilocular. Reaspiration was performed in six patients. Two of the latter required needle aspiration three times, both of these patients had matted nodes with multilocular collections. One of these patients underwent excision revealing necrotic caseous material in the nodes.

Regression rate in the study group two, four, and six months after needle aspiration was significantly higher compared with the control group (table). No recurrence was observed up to six months after healing. Spontaneous drainage with development of sinus tract formation was also markedly less in the study group compared with the control group four and six months after needle aspiration (table).

Size of the node $(1 \cdot 5-5 \mathrm{~cm})$, age of the patient, and time interval after vaccination did not influence the outcome. However, 24 fluctuant nodes in the study group regressed, on average, in a shorter period of time than non-fluctuant ones $(6.7 v 11.8$ weeks) Cytological study mainly revealed necrosis, neutrophils, a few plasma cells, lymphocytes, foreign body giant cells, and epithelioid cells.

\section{Discussion}

Enlargement of the regional nodes after BCG vaccination is actually a form of immune response and is only secondary to invasion of the nodes by a non-virulent strain in the vaccine. The chance of this complication occurring is related to the type of vaccine, dose, concentration, and route (depth) of administration (subcutaneous $v$ intradermal). ${ }^{4}$ Immunologically normal newborns have double the incidence of adenitis compared with older infants and children. ${ }^{4}$ 
Rate of regression and spontaneous drainage in 77 patients

\begin{tabular}{|c|c|c|c|}
\hline & \multicolumn{3}{|l|}{ Time (months) } \\
\hline & 2 & 4 & 6 \\
\hline $\begin{array}{l}\text { No }(\%) \text { regressed } \\
\text { Study group } \\
\text { Control group } \\
95 \% \mathrm{CI}\end{array}$ & $\begin{array}{l}25(58)^{\star} \\
3(9)^{\star} \\
0.403 \text { to } 0.583\end{array}$ & $\begin{array}{l}38(88) \dagger \\
9(26) \dagger \\
0.529 \text { to } 0.709\end{array}$ & $\begin{array}{l}41(95) \ddagger \\
22(65) \ddagger \\
0 \cdot 218 \text { to } 0.394\end{array}$ \\
\hline $\begin{array}{l}\text { No (\%) spontaneous drainage } \\
\text { Study group } \\
\text { Control group } \\
95 \% \mathrm{CI}\end{array}$ & $\begin{array}{l}3(7) \oint \\
5(15) \oint \\
-0.21 \text { to } 0.06\end{array}$ & $\begin{array}{l}3(7) \text { व } \\
13(38) \\
0.129 \text { to } 0.495\end{array}$ & $\begin{array}{l}3(7) \| \\
15(44) \| \\
0.188 \text { to } 0.555\end{array}$ \\
\hline
\end{tabular}

$\mathrm{CI}=$ Confidence interval.

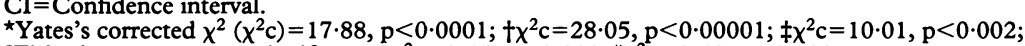
§Fisher's exact test, not significant; $\llbracket \chi^{2} c=9.45, p<0.003 ; \| \chi^{2} c=9.63, p<0.002$.

Although the natural course of BCG adenitis, whether suppurative or not, is ultimate regression, different modes of management have been suggested and tried. ${ }^{13}$ Medical (systemic) treatment, though it may be indicated in some cases, ${ }^{12}$ is not recommended routinely. Excision and drainage, formerly advised as the first step in the management of these patients, ${ }^{5}$ now seems to be futile. Total excision, which has also been advised in rapidly developing adenitis (less than two months after vaccination), ${ }^{1}$ or if the node diameter is greater than $3 \mathrm{~cm},{ }^{2}$ should be reserved for those cases in whom other less aggressive procedures have not been successful. However, if left intact, the enlarged nodes need several months to regress.

This prospective study has shown that nodes after needle aspiration, especially if fluctuant, regress in a much shorter period of time and the chance of sinus tract formation is dramatically reduced. Çaglayan et al have also obtained satisfactory results in non-drained suppurative adenitis after needle aspiration. ${ }^{3}$

This phenomenon can be explained on the basis of findings in the caseous (necrotic) material obtained from experimental pulmonary tuberculosis in animal models. ${ }^{6}$ The necrotic tissue thus formed by a delayed hypersensitivity reaction is related to the local concentration of tuberculin antigens secondary to increased numbers of the extracellular multiplied bacilli. This medium contains various inflammatory mediators, including cytokines, arachidonic acid metabolites, reactive oxygen, and hydrolytic enzymes produced by the host (dead microbicidal macrophage) or probably tuberculosis bacilli. ${ }^{6}$ Furthermore, an anoxic environment with low $\mathrm{pH}$, containing toxic fatty acids, is also produced. Because of the similarities in the histological picture in this situation and those seen in post-BCG adenitis, the inflammatory mediators and chemical contents may, at least to some extent, be comparable in both conditions. Conclusively, once the liquified necrotic caseous material (pus) containing detrimental substances is drained, spontaneously or by needle aspiration, node regression should logically be enhanced.

Lack of response to needle aspiration may be due to inadequate evacuation of the nodes or severity of delayed hypersensitivity, as well as cellular immunity response, causing multiple node enlargement associated with solid (unliquified) necrosis, thus delaying the healing process. In case of recollection, reaspiration is usually all that is necessary. If there is no response after the third attempt, the nodes should be excised.

In conclusion, needle aspiration has a major role in the management of post-BCG adenitis, particularly if it is fluctuant or suppurative. Not only does it prevent spontaneous drainage, but also facilitates node regression in a relatively short period of time. If properly done, it has no significant complication or morbidity. Surgery should be reserved for those with repeated collections after needle aspiration, especially if the nodes are matted and multilocular.

We are grateful to Dr $\mathrm{H}$ Mousavi for his invaluable contribution in this research by performing sonography, and Dr P V Kumar for his excellent cytological studies.

1 Caglayan S, Yegin O, Kayran $\mathrm{K}$, et al. Is medical therapy effective for regional lymphadenitis following BCG vaccination. $A m$ f $D$ is $C$ hild $1987 ; 141: 1213-4$

2 Oguz F, Müjgan S, Alper G, et al. Treatment of Bacillus Calmette-Guerin-associated lymphadenitis. Pediatr Infect Dis $\mathcal{f} 1992 ; 11: 887-8$.

3 C.aglayan S, Arikan A, Yaprak I, et al. Management of suppuration in regional lymph nodes secondary to BCG vaccination. Acta Paediatr Fpn 1991; 33: 699-702.

4 Milstien JB, Gibson JJ. Quality control of BCG vaccine by WHO: a review of factors that may influence vaccine effectiveness and safety. Bull World Health Organ 1990; 68: 93-108.

5 Tam PKH, Stroebel AB, Saing $\mathrm{H}$, et al. Caseating regional lymphadenitis complicating BCG vaccination: a report of 6 cases. Arch Dis Child 1982; 57: 952-4.

6 Dannenberg AM Jr. Immunopathogenesis of pulmonary tuberculosis. Hospital Practice 1993; 15: 33-40. 